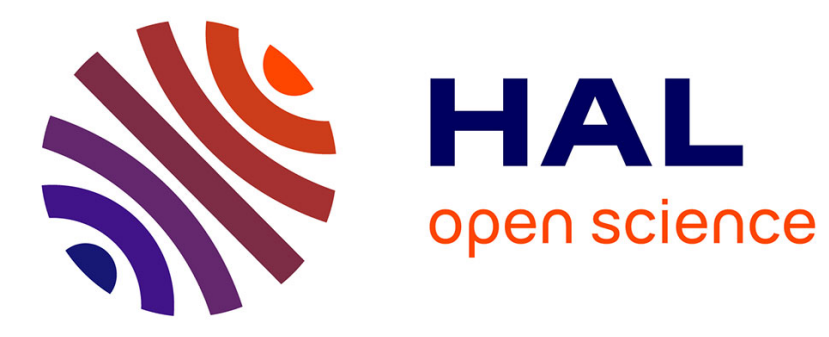

\title{
Trapping and release of helium in tungsten
}

Pierre-Emile Lhuillier, Taieb Belhabib, Pierre Desgardin, Blandine Courtois, Thierry Sauvage, Marie-France Barthe, Anne-Lise Thomann, Pascal Brault, Yves Tessier

\section{- To cite this version:}

Pierre-Emile Lhuillier, Taieb Belhabib, Pierre Desgardin, Blandine Courtois, Thierry Sauvage, et al.. Trapping and release of helium in tungsten. Journal of Nuclear Materials, 2011, 416, pp.13-17. 10.1016/j.jnucmat.2010.12.042 . hal-00642263

\section{HAL Id: hal-00642263 https://hal.science/hal-00642263}

Submitted on 18 Apr 2012

HAL is a multi-disciplinary open access archive for the deposit and dissemination of scientific research documents, whether they are published or not. The documents may come from teaching and research institutions in France or abroad, or from public or private research centers.
L'archive ouverte pluridisciplinaire HAL, est destinée au dépôt et à la diffusion de documents scientifiques de niveau recherche, publiés ou non, émanant des établissements d'enseignement et de recherche français ou étrangers, des laboratoires publics ou privés. 
Trapping and release of helium in tungsten

Pierre-Emile Lhuillier ${ }^{1}$, Taïeb Belhabib ${ }^{1}$, Pierre Desgardin ${ }^{1}$, Blandine Courtois ${ }^{1}$, Thierry Sauvage ${ }^{1}$, MarieFrance Barthe ${ }^{1}$, Anne-Lise Thomann ${ }^{2}$, Pascal Brault ${ }^{2}$, Yves Tessier ${ }^{2}$

CNRS, UPR3079 CEMHTI, 1D Avenue de la Recherche Scientifique, 45071 Orléans Cedex 2, France

Université d'Orléans, Faculté des Sciences, Avenue du Parc Floral, BP 6749, 45067 Orléans Cedex 2,

France

GREMI Polytech'Orléans, 14 rue D’Issoudun, BP 6744, 45067 Orléans Cedex 2, France

\section{Abstract}

The behavior of tungsten under irradiation and helium implantation is a major stake of the materialrelated issues of fusion reactors. In this perspective the fate of helium in tungsten was studied by mean of several characterization techniques. The aim of this study is to highlight the trapping mechanisms of helium in tungsten and their correlation with implantation-induced defects. Helium was implanted into tungsten at two different energies, 0.32 and $60 \mathrm{keV}$. The helium was studied as a function of temperature by using nuclear reaction analysis. The migration propensity of helium was correlated with the release rate of helium after annealing at a given temperature. In addition, the helium trapping sites and evolution with post-implantation annealing was investigated using Doppler-Broadening Positron Annihilation Spectroscopy. It has been shown that the release, and so the migration, of helium is guided by the concentration of implantation-induced defects and the nature of the helium traps created during the implantation.

\section{Introduction}

In the field of the design of the future thermonuclear fusion reactor, the issue of materials science is a major stake. In the future experimental thermonuclear reactor ITER, the first wall materials will have to bear extremely severe conditions. In particular, the shielding material of the divertor, an essential component which exhausts the dust particles of the plasma, will be submitted to most hostile conditions. Tungsten is the best candidate for the shielding material of the divertor. Tungsten is also considered as first wall armor in DEMO, the fusion reactor that will be built next after ITER to demonstrate the feasibility of energy production. In operation, tungsten will be submitted to a high neutron irradiation. Indeed the fusion reaction between deuterium and tritium releases a highly energetic $14 \mathrm{MeV}$ neutron which induces atom displacement in the structure material of the reactor. Moreover, the first wall materials and thus the tungsten are submitted to the bombardment of the plasma particles [1] and to implantation of light atom created by nuclear transmutation [2] due to neutron irradiation. The plasma particles bombardment induces the implantation of helium and hydrogen in the near surface of the tungsten and can severely damage the material. Consequently the understanding of

* Corresponding author at: CNRS, UPR3079 CEMHTI, 1D Avenue de la Recherche Scientifique, 45071 Orléans Cedex 2, France

E-mail address: marie-France.barthe@cnrs-orleans.fr (M.F. Barthe). the behavior of tungsten in fusion environment is a major issue of the material-related stake of fusion reactors.

On the one hand, to address this issue, many studies focus on ITER-like implantation or irradiation conditions to estimate the fate of the materials. In this option, the helium implantation flux should be of the order of $10^{20} \mathrm{~cm}^{-2} \mathrm{~s}^{-1}$ [3], in order to fit with the particle flux predicted for ITER. On the other hand, some experimental works - among which the present work is found - focus on the fundamental properties of the tungsten under irradiation and implantation $[4,5]$ and coupling the data with numerical simulation $[6,7]$. This outlook requires investigating the material properties submitted to rather low implantation flux and fluence, to focus on the early stages of the atomic processes. The final aim of this approach is to get a predictive tool of the behavior and lifetime of tungsten components in fusion environment. In this perspective, this paper investigates the behavior of helium implanted in tungsten with respect to different implantation conditions. Several of our results gave rise to important properties of irradiation defects and helium in tungsten. In the field of defects, the nature of the defects induced by the implantation of helium ions at $800 \mathrm{keV}$ was found to be solely tungsten single vacancies [8]. With the use of Positron Annihilation Spectroscopy (PAS) the thermal behavior of the tungsten vacancy was found. In particular, the tungsten monovacancy was shown to be mobile from $\sim 473 \mathrm{~K}[8]$ and thus induces the creation of vacancy clusters. Concerning the behavior of tungsten, it was demonstrated that helium ions implanted at energy of the order of $500 \mathrm{keV}$ are deeply trapped in the tungsten matrix [9]. 
Indeed the helium mobility only at short range was observed with the evolution of the helium depth profile vs. temperature. Thus the helium implanted in tungsten at $500 \mathrm{keV}$ does not exhibit any long range migration up to $1773 \mathrm{~K}$

Thus the aim of the present work is to draw a map of the trapping phenomenon of helium in tungsten in some specific conditions, with respect to the implantation conditions and the trapping defect creation. In particular the trapping of helium was characterized by the propensity of helium to be released from the material at a given temperature. Taking into account the previous results, it is possible to give some clues about the main parameters controlling the helium trapping in the investigated conditions.

\section{Experimental}

The material used in this study was polycrystalline tungsten with a purity of $99.95 \%$ wt. The samples were $7 \times 7 \mathrm{~mm}^{2}$ in size and $150 \mu \mathrm{m}$ thick and came from laminated tungsten foils. In order to remove the native defects of the material, the samples were submitted to a thermal treatment at $1873 \mathrm{~K}$ during $1 \mathrm{~h}$ under high vac uum $\left(10^{-6}-10^{-7} \mathrm{mbar}\right)$. Consecutively to the thermal treatment the quality of the sample was reported in another study [8]. With the use of Positron Annihilation Spectroscopy (PAS) it was found that a large part of the pre-existing defects were removed from the material. The grain size also increases during annealing to an average value higher than $10 \mu \mathrm{m}$. Thus the initial state of the samples prior to implantation can be considered as almost "defectfree" as regard to the detection limit of the PAS technique.

The trapping behavior of helium in tungsten was investigated with two different implantation conditions. On the one hand, one set of sample was implanted with low energy helium ions a $0.32 \mathrm{keV}$. The implantation was performed with the use of a plasma process. The samples were immersed is the periphery of a RF inductively coupled ${ }^{3} \mathrm{He}$ plasma $\left(2 \times 10^{-5} \mathrm{mbar}, 50 \mathrm{~W}\right)$. The sample holder was submitted to a biased voltage of $300 \mathrm{~V}$ so that the helium ions were accelerated though the sheath. Taking into account the plasma potential $\left(V_{p}=20 \mathrm{~V}\right)$ the energy of the incident ions was $0.32 \mathrm{keV}$. The power load applied to the plasma was low enough that only monocharged helium ions were created in the plasma. The samples were immersed in the plasma under a mean ${ }^{3} \mathrm{He}^{+}$flux of $2 \times 10^{14} \mathrm{~cm}^{-2} \mathrm{~s}^{-1}$ and with incident helium fluence of the order of $10^{18} \mathrm{~cm}^{-2}$. On the other hand, another set of samples was implanted with helium ions at $60 \mathrm{keV}$. The implantations were performed with an ion implantor and several ion fluences from $2 \times 10^{13}$ to $10^{16} \mathrm{~cm}^{-2}$ were investigated. During the implantation the temperature of the sample remained below $333 \mathrm{~K}$. In the above conditions, the implanted helium profile was simulated with the SRIM program. It especially enables to estimate the mean implantation depth of helium. Nevertheless, one should mention that, since SRIM considers the sample lattice to be amorphous, the simulated helium depth distribution could exhibit some slight differences with the experimental one.

After implantation the behavior of helium was investigated by means of nuclear reaction analysis (NRA). This ion beam analysis technique is based on the ${ }^{3} \mathrm{He}\left(\mathrm{d},{ }^{1} \mathrm{H}\right), \alpha$ nuclear reaction. The analysis was performed on a dedicated apparatus called DIADDHEM a the CEMHTI Cyclotron in Orleans France [10]. The probing deuterium beam with energy of $750 \mathrm{keV}$ is focused on the sample. The number of protons generated by the nuclear reaction which escape from the backside of the sample are detected. It allows determining the absolute amount of helium which remains in the sample. A detailed description of the technique has been reported in [11]

The nature of the trapping site of helium was examined with the use of Positron Annihilation Spectroscopy. The samples have been characterized by using a Doppler Broadening Spectrometer coupled with the slow positron beam available at the CEMHTI laboratory. This set up enables to implant positrons in the samples with variable energy ranging from 0.5 to $25 \mathrm{keV}$. In the tungsten, it corresponds to a maximum probed layer of $\sim 700 \mathrm{~nm}$. The positrons implanted in the samples lose their kinetic energy and are thermalised. Then they annihilate with an electron of the sample. The annihilation of the positron/electron pair will generate the emission of $2 \gamma$ photons with energy of $511 \mathrm{keV} \pm \Delta E$. The $\Delta E$ quantity is the Doppler shift of the annihilation photon and is proportional to the kinetic momentum of the annihilation electron in the direction of propagation of the $511 \mathrm{keV}$ photon. The Doppler Broadening spectrum of the annihilation photon is recorded and gives an image of the distribution of the kinetic momentum of the electron in the sample. We thus have access to the knowledge of the electronic density experienced by the positron at its annihilation site. One should note that, due to its positive charge, the positron is especially trapped by the low electron density region of the sample, especially the vacancy-type defects present in the sample. Thus the PAS technique is particularly sensitive to the nature and relative concentration of vacancy-type defects in the material. The Doppler broadening of the annihilation spectrum has been characterized by the $S$ and $W$ integral parameters. $S$ (resp. $W$ ) represents the annihilation fraction with low (resp. high) momentum electron and is thus related with annihilation fraction with valence (resp. core) electrons. The accurate definition of $S$ and $W$ can be found in [5]. When the material contains vacancy defects $S$ increases and $W$ decreases and each annihilation state is characterized with a specific $S, W$ values.

\section{Results}

\section{1. $60 \mathrm{keV} \mathrm{He}$ implantation}

The implantation profile of helium ions at $60 \mathrm{keV}$ was calculated with the SRIM software [12]. The implantation depth range of the helium atoms is located around $130 \mathrm{~nm}$. Four different implantation fluences were used and the effective fluence after implantation was measured. The comparison of the theoretical fluence and the effectively measured fluence is shown in Table 1 . The table also displays the level of displacement per atom (dpa) induced by the incident ion. This value, calculated by SRIM, does not take into account the recombination and annihilation of Frenkel pair at room temperature, thus the value should be taken as the upper limit of the effective displacement concentration in the

\section{Table 1}

Implantation parameters of the ${ }^{3} \mathrm{He} 60 \mathrm{keV}$ implantation in tungsten. The effective fluence and the helium released were measured by mean of nuclear reaction analysis. The damage level was calculated by SRIM and represents the number of tungsten atoms displaced by the incident ions (in dpa, displacement per atom).

\begin{tabular}{lllll}
\hline Nominal fluence $\left(\mathrm{cm}^{-2}\right)$ & Effective fluence $\left(\mathrm{cm}^{-2}\right)$ & $\begin{array}{l}\text { Damage (calculated } \\
\text { by SRIM) (dpa) }\end{array}$ & $\begin{array}{l}\text { Helium concentration } \\
\text { at } 130 \mathrm{~nm} \text { (at.\%) }\end{array}$ & $\begin{array}{l}\text { Helium released fraction } \\
\text { at } 1873 \mathrm{~K}(\%)\end{array}$ \\
\hline $2 \times 10^{13}$ & $1.89 \pm 0.30 \times 10^{13}$ & $\sim 4 \times 10^{-4}$ & $2 \times 10^{-3}$ & $75 \pm 5$ \\
$10^{14}$ & $1.16 \pm 0.12 \times 10^{14}$ & $\sim 2 \times 10^{-3}$ & 0.01 & $54 \pm 5$ \\
$10^{15}$ & $1.21 \pm 0.12 \times 10^{15}$ & $\sim 0.02$ & 0.1 & $10 \pm 5$ \\
$10^{16}$ & $\sim 1.1 \times 10^{16}$ & $\sim 0.2$ & 1 & $0 \pm 5$ \\
\hline
\end{tabular}


material. One can expect that implantation defects are mainly single vacancies $[8,9]$ which are not mobile at room temperature [8]. In the region where the helium atom are implanted the helium to displacement ratio is thus of the order of 0.05 He per vacancy. Even if the binding energy of interstitial helium atoms together is rather high [7] and could lead to the creation of highly stable interstitial helium clusters, the helium to damage ratio (of the order of $1 \mathrm{He}$ for 20 implantation-induced single vacancies) suggest that, during implantation, the main trapping sites of helium are the single vacancies. Table 1 gives the fraction of helium released from the sample after annealing at $1873 \mathrm{~K}$ during $1 \mathrm{~h}$ as a function of the implantation fluence. Owing that the helium is located at a mean depth of $130 \mathrm{~nm}$, the desorption rate give an information about the migration and trapping propensity of helium in tungsten. The desorption rate at $1873 \mathrm{~K}$, highly depends on the initial implantation fluence. Indeed no desorption of helium is observed for the highest fluence $\left(10^{16} \mathrm{~cm}^{-2}\right)$ whereas the released fraction of helium at $1873 \mathrm{~K}$ reaches $75 \pm 5 \%$ for the lowest initial fluence $\left(2 \times 10^{13} \mathrm{~cm}^{-2}\right)$.

For the highest implantation fluence, $10^{16} \mathrm{~cm}^{-2}$, the PAS measurement of samples after implantation and after post-implantation annealing (at $1873 \mathrm{~K}$ ) are available. The $S$ profile as a function of the positron energy is plotted in Fig. $1 \mathrm{a}$ and $S$ vs. $W$ data are displayed in Fig. 1b. On Fig. 1b is also displayed the point which corresponds to the $S(W)$ annihilation characteristics of the virgin sample. This value has been obtained by the refinement of the experimental data extracted with the use of fitting software (VEPFIT, see details in Ref. [13]). This point is assimilated to the initial and reference state of the "defect-free" tungsten samples. In addition to the $S(W)$ data profiles (Fig. 1b), the points which correspond to the annihilation parameters of the region of interest around $130 \mathrm{~nm}$ for the two profiles (also obtained with the VEPFIT software) are specifically high lighted. Fig. 1b also displays line D, which joints the initial state of the samples and the point characteristic of the tungsten monovacancy. One can observe that the point corresponding to the region at $130 \mathrm{~nm}$ of the as-implanted sample is just below line D. This corresponds to the presence of vacancy-type defects with inne electron density lower than the inner density of tungsten monovacancy. At $60 \mathrm{keV}$, a high density of tungsten vacancies is created concomitantly to the helium implantation. After implantation, the PAS data at $130 \mathrm{~nm}$ can be interpreted as the presence of monovacancies $V$ and helium-filled vacancies. The helium should thus be located in $x \mathrm{He} \cdot n \mathrm{~V}$ clusters of very small size $(n \approx 1)$ and with helium to vacancy $(x / n)$ ratio close to 1 .

After annealing at $1873 \mathrm{~K}$, the $S(E)$ profile has drastically changed. The $S$ value at the incident energy of the positrons of $13 \mathrm{keV}$ which corresponds to the mean implantation depth of $130 \mathrm{~nm}$ (point at $\sim 13 \mathrm{keV}$ ) has severely increased compared to the as-implanted sample. The corresponding point of the $S(W)$ diagram appears to be located well above line D. It indicates that the mean free volume of the defects has increased with annealing. Since $100 \pm 5 \%$ of the implanted helium still remains in the sample (see Table 1), one could expect that the helium is trapped at heliumfilled cavities.

\section{2. $0.32 \mathrm{keV} \mathrm{He}$ implantation}

The low energy helium implantation - at $0.32 \mathrm{keV}$ - leads to helium introduction in the very near surface of the sample in the 30 first nanometers under the surface. The effective helium implanted in the sample was measured and is equal to $\sim 7 \times 10^{15} \mathrm{He} / \mathrm{cm}^{2}$. The helium concentration in the near surface region is of the order of 4 at.\%. Moreover the implantation energy of $0.32 \mathrm{keV}$ is below the displacement threshold in $W$. Indeed the maximum energy which could be transferred from an incident ${ }^{3} \mathrm{He}$ ion of $0.32 \mathrm{keV}$ to the atoms of the tungsten matrix is of the order of $19 \mathrm{eV}$. Owing that the displacement threshold energy of atom in the tungsten lattice is $90 \mathrm{eV}$ [14], one could expect that the incident ions will not induce displacement in the tungsten lattice by elastic collision. The mobility and trapping of helium were also investigated by measuring the helium released fraction after annealing at $1873 \mathrm{~K}$ during $1 \mathrm{~h}$ under vacuum. It was found that the released fraction reaches $90-100 \%$ at $1873 \mathrm{~K}$. In order to identify the nature of the helium trapping site, the samples implanted at low energy were characterized by PAS. The $S(E)$ profile of the sample implanted at $0.32 \mathrm{keV}$ at the fluence of $7 \times 10^{15} \mathrm{~cm}^{-2}$ was measured and is displayed in Fig. 1. The PAS data of the helium implanted region (in the very near surface of the sample) was extrapolated with the VEPFIT software and was found to be

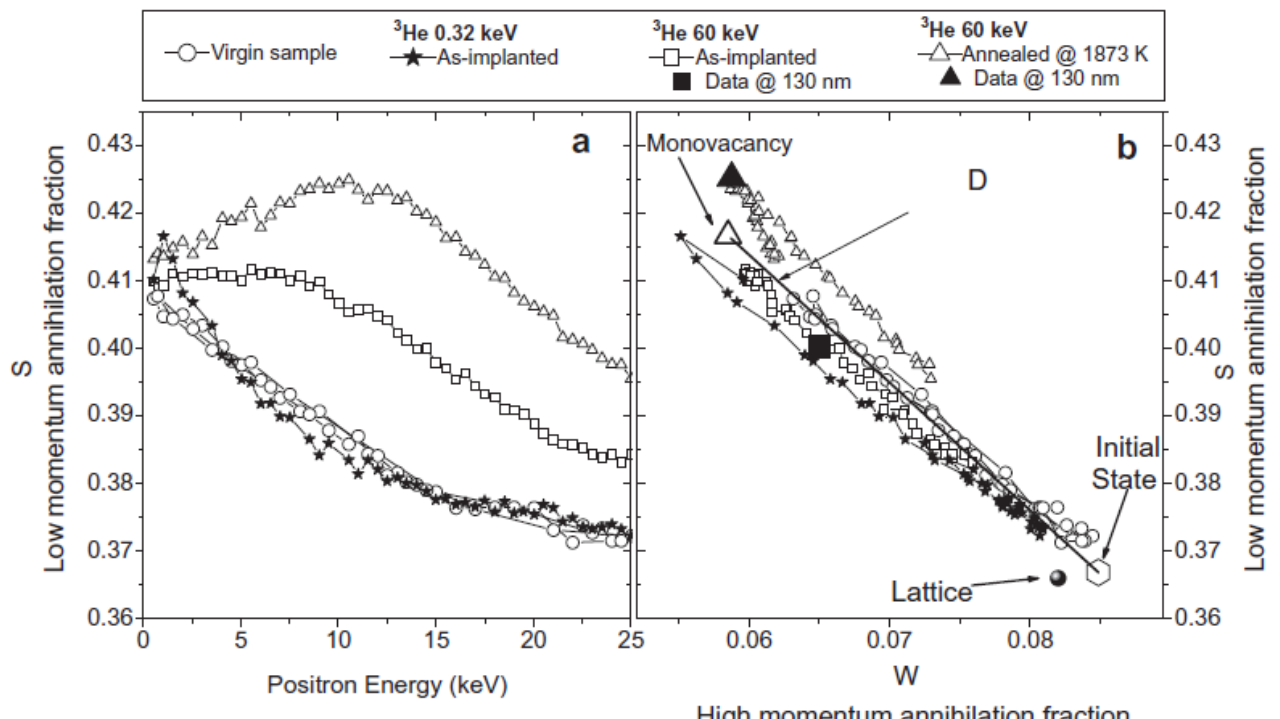

High momentum annihilation fraction

Fig. 1. Positron annihilation characteristics of the tungsten samples implanted with ${ }^{3} \mathrm{He}$ at $60 \mathrm{keV}$ and at $0.32 \mathrm{keV}$. (a) $S$ vs. incident positron energy (b) $S$ vs. $W$. $S$ (resp. $W$ ) represents the annihilation fraction of positron/electron pair with low (resp. high) kinetic momentum. 


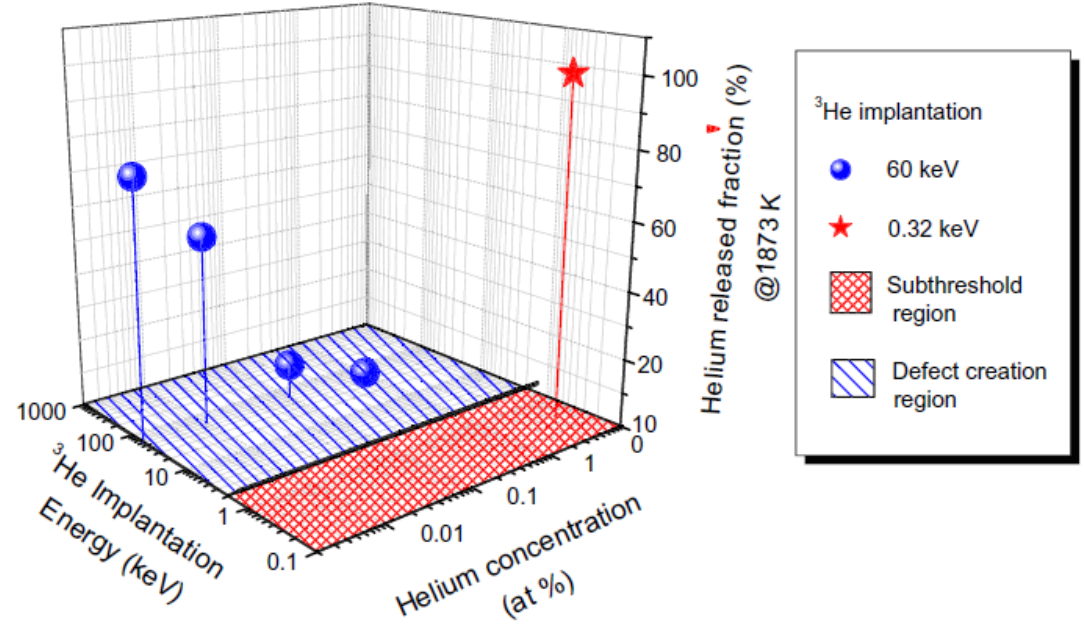

Fig 2. Helium released fraction after annealing at $1873 \mathrm{~K}$ during $1 \mathrm{~h}$ under vacuum as a function of the implantation energy of ${ }^{3} \mathrm{He}$ ions and as a function of the maximum helium concentration (calculated by SRIM). The implantation was performed on tungsten samples annealed at high temperature (see text) before implantation and the release rate of helium at $1873 \mathrm{~K}$ was measured by nuclear reaction analysis.

$S=0.447$ and $W=0.028$ (not displayed in Fig. $1 \mathrm{~b}$ for the readability of the graph). This point is far below line $\mathrm{D}$ with very high $S$ value and low $W$ value. It suggests the presence in the region of implantation of the helium atoms of a dense array of helium-filled vacancy-clusters. Taking into account the position of the point which characterizes the annihilation parameters of the helium implanted region far below line D, we suggest that the defects detected by the PAS technique are $x$ He. $n V$ complexes with high helium to vacancy ratio. The interpretation of the data is based on the theoretical calculation of Toyama et al. [15]. The authors performed theoretical simulation of the Doppler broadening behavior of helium-filled vacancy clusters in pure bcc iron as a function of the helium to vacancy ratio. For a given vacancy cluster of size $V_{n}$, the increase of the number $x$ of helium atoms inside the $x \mathrm{He} \cdot n \mathrm{~V}$ complex induces a simultaneous decrease of the $S$ and $W$ parameters. In those implantation conditions, the helium could effectively be trapped in $x \mathrm{He} \cdot n \mathrm{~V}$ complexes created by the mutation phenomenon [16]. This mechanism consists of the ejection of a self-interstitial atom (SIA) of tungsten by a $x \mathrm{He} \cdot n \mathrm{~V}$ complex which absorbs atoms of helium. It must be noted that no evidence of blistering was observed on those samples either by scanning electron microscopy or transmission electron microscopy.

The helium released fractions measured after annealing at $1873 \mathrm{~K}$ are summarized on a 3D plot on Fig. 2. The plot is split in two regions denoted as subthreshold region and defect creation region. The boundary between both those regions corresponds to the maximum implantation energy of ${ }^{3} \mathrm{He}$ ions below which no defects are created by elastic collision during implantation. The maximum recoil energy $E_{\max }$ transmitted by the ${ }^{3} \mathrm{He}$ ion of energy $E$ to the tungsten target atom is given by the relation [17]:

$E_{\max }=0.063 E$

When $E_{\max }$ is over the displacement threshold energy in tungsten $(90 \mathrm{eV})$ the implantation generates defects such as Frenkel pairs. With ${ }^{3} \mathrm{He}$ ion, $E_{\max }$ is equal to $90 \mathrm{eV}$ when the incident energy is about $1.4 \mathrm{keV}$.

\section{Discussion}

At $60 \mathrm{keV}$, one can expect that implantation defects are mainly single vacancies $[8,9]$ which are not mobile at room temperature
[8]. Since self-interstitial atoms (SIAs) are mobile at temperature well below room temperature [18], a large fraction of the SIAs created by implantation is expected to recombine with vacancy or vanish from the matrix. The SIA also exhibits a high binding energy to helium atom, $E_{b}=0.94 \mathrm{eV}$ [19]. Some SIAs may survive the recombination processes since they are trapped at helium atoms. The above binding energy must be compared to the binding energy of He with a single vacancy, $E_{b}=4.57 \mathrm{eV}$ which corresponds to a dissociation temperature around $1520 \mathrm{~K}$ [20]. The dissociation of He from SIA should occur at temperature well below $1520 \mathrm{~K}$. It has been shown elsewhere, that, in those conditions, the release of helium begins at $1473 \mathrm{~K}$ and is due to the dissociation of $\mathrm{He}$ from single helium-vacancy He.V. Since no desorption stage below $1473 \mathrm{~K}$ was observed [21], it suggests the He detrapped from SIA are immediately trapped by the free single vacancy available in the sample. Eventually the SIAs do not seem to play a significant role in the trapping of helium at the temperature investigated here.

At $1873 \mathrm{~K}$, the fraction of released helium highly depends on the implantation fluence. As we have seen before, the helium desorption decreases when the implantation fluence increases. It indicates that the efficiency of the helium trapping increases with the implantation fluence. Moreover it was shown by Becquart and Domain [19] that the binding energy of a helium atom to a $x$ He. $n V$ decreases when the $x / n$ ratio increases. It can be suggested that the increase of fluence induces the trapping of helium at $x$ He $n V$ clusters with lower $x / n$ ratio. When the implantation fluence increases, the probability for an implantation-induced single vacancy to be created in the nearest neighbor site of a pre-existing vacancy becomes high. It has to be noticed that Becquart and Domain have calculated that the binding energy of the tungsten divacancy is negative [22]. On the contrary, they have also shown that the binding energy of a vacancy to a helium-filled vacancy $\mathrm{HeV}$ becomes slightly positive ( $\left.E_{b}=0.07 \mathrm{eV}[19]\right)$. Thus the increase of the fluence should promote the creation of $\mathrm{He} \cdot n \mathrm{~V}(n>1)$ complexes during the implantation. Since the binding energy of helium atom with a He.nV clusters increases with $n$, the increase of the implantation should deepen the initial (just after implantation) average trapping of helium atoms in the matrix of tungsten. Moreover, during annealing, the monovacancies become mobile, above $\sim 473 \mathrm{~K}$ [8]. The clustering of the mobile vacancies should induce the crea- 
tion of larger $x \mathrm{He} \cdot n \mathrm{~V}$ clusters. The PAS results have effectively shown the creation of helium-filled cavities. Since the monovacancy initial concentration calculated from SRIM is significantly higher than the helium concentration available in the material, the clustering of monovacancies should leads to the decrease of the $x / n$ ratio of the resulting $x \mathrm{He} \cdot n \mathrm{~V}$ clusters. As a consequence, the trapping of helium becomes deeper and deeper when the vacancy clustering is operational. It can be expected that the complexes should be so much bigger that the concentration of mobile vacancies is high that is to say that the initial implantation fluence is high. This interpretation could explain the inhibition of the desorption at $1873 \mathrm{~K}$ observed with the increase of the implantation fluence on the samples implanted at $60 \mathrm{keV}$.

In the case of the low energy implantation $(0.32 \mathrm{keV})$, we have seen in the above results that helium atoms are initially in highly pressurized $x$ He $\cdot n \mathrm{~V}$ clusters $(x / n \gg 1)$. Even if the helium could partially remain in interstitial position, the vacancy-type defects detected by PAS suggest that a significant fraction of helium is trapped in helium-filled vacancy-type defects. After annealing at $1873 \mathrm{~K}$, the whole helium has been released from the sample. Moreover it was shown elsewhere that the major part of the release (up to more than $65 \%$ ) occurs below $1473 \mathrm{~K}$ [23]. Helium atoms are thus very shallowly bounded to their trapping sites. The helium atoms could then be easily detrapped from the $x$ He $n \mathrm{~V}$ because of the annealing temperature.

\section{Conclusion}

It has been shown that the correlation between PAS data and the measurement of the released fraction of helium at $1873 \mathrm{~K}$ en ables to discuss the nature of the trapping sites for helium introduced in different implantation conditions. The data suggest that in displacement subthreshold conditions (implantation energy of $0.32 \mathrm{keV}$ ), helium is trapped in highly pressurized helium-vacancy complexes with high helium to vacancy ratio. During the annealing, the helium atoms are easily detrapped from those clusters and the desorption of helium reaches $90-100 \%$ at $1873 \mathrm{~K}$. With the implantation at $60 \mathrm{keV}$, the helium atoms are initially trapped at $x \mathrm{He} \cdot n \mathrm{~V}$ complexes surrounded with a high concentration of single vacancies. During annealing, the mobility (from $\sim 473 \mathrm{~K}$ ) and clustering of single vacancies induces the growth of the $x \mathrm{He} \cdot n \mathrm{~V}$ complexes. The $x / n$ ratio of the resulting traps should be so much low that the initial monovacancy concentration is high. This phenomenon allows to explain the inhibition of the helium release with the increase of the implantation fluence observed in the samples implanted at $60 \mathrm{keV}$. Eventually it has been shown that the release, and so the migration, of helium after annealing is controlled by two main parameters which are the concentration of trapping site and the helium to vacancy ratio of the trapping complexes created during implantation.

\section{Acknowledgements}

The study was partially supported by the Interdisciplinary Program for Energy (PIE) of CNRS - France. This work was carried out within the framework of the European Fusion Development Agreement and the French Research Federation for Fusion Studies. It is supported by the European Community under the Contract of association between Euratom and CEA. The views and opinions expressed herein do not necessarily reflect those of the European Commission.

\section{References}

[1] H. Bolt, V. Barabash, G. Federici, J. Linke, A. Loarte, J. Roth, K. Sato,J. Nucl. Mater 307 (2002) 43-52.

[2] R. Behrisch, V. Khripunov, R.T. Santoro, J.M. Yesil, J. Nucl. Mater. 258-263 (1998) 686-693.

[3] H. Bolt, V. Barabash, W. Krauss, J. Linke, R. Neu, S. Suzuki, N. Yoshida, ASDEX Upgrade Team, J. Nucl. Mater. 329-333 (2004) 66-73.

[4] H.T. Lee, A.A. Haasz, J.W. Davis, R.G. Macaulay-Newcombe, D.G. Whyte, G.M. Wright, J. Nucl. Mater. 363-365 (2007) 898-903.

5] A. Debelle, M. Barthe, T. Sauvage, J. Nucl. Mater. 376 (2008) 216-221.

[6] S. Sharafat, A. Takahashi, Q. Hu, N.M. Ghoniem, J. Nucl. Mater. 386-388 (2009) 900-903.

7] CS. Becquart, C. Domain, Phys. Rev. Lett. 97 (2006) 196402-196404.

[8] P. Lhuillier, M. Barthe, P. Desgard in, W. Egger, P. Sperr, Phys. Status Solidi (c) 6 (2009) 2329-2332

[9] A. Debelle, M. Barthe, T. Sauvage, R. Belamhawal, A. Chelgoum, P. Desgardin, H. Labrim, J. Nucl. Mater. 362 (2007) 181-188.

[10] T. Sauvage, H. Erramli, S. Guilbert, L. Vincent, M.-F. Barthe, P. Desgardin, C Blondiaux, C. Corbel, J.P. Piron, F. Labohm, A. Van Veen, J. Nucl. Mater. 327 (2004) 159-164.

[11] F. Pászti, Nucl. Instrum. Methods Phys. Res., Sect. B 66 (1992) 83-106.

12] J. Ziegler, J. Biersack, U. Littmark, The Stopping and Range of lons in Solids, Pergamon, New York, 1985.

[13] A. van Veen, H. Schut, M. Clement, J.M.M. de Nijs, A. Kruseman, M.R. Ijpma, Appl. Surf. Sci. 85 (1995) 216-224.

[14] Standard Practice for Neutron Radiation Damage Simulation by ChargeParticle Irradiation, Philadelphia, 1996.

[15] T. Toyama, Z. Tang, M. Eldrup, Y. Nagai, P. Jung, M. Hasegawa, B.N. Singh, personnel communication.

[16] H. Iwakiri, K. Yasunaga, K. Morishita, N. Yoshida, J. Nucl. Mater. 283-287 (2000) 1134-1138.

[17] J. Amano, D.N. Seidman, J. Appl. Phys. 56 (1984) 983-992

[18] S. Okuda, H. Mizubayashi, Phys. Rev. Lett. 34 (1975) 815-817.

[19] C.S. Becquart, C. Domain, J. Nucl. Mater. 385 (2009) 223-227.

[20] E.V. Kornelsen, A.A. Van Gorkum, J. Nucl. Mater. 92 (1980) 79-88.

21] A. Debelle, P. Lhuillier, M. Barthe, T. Sauvage, P. Desgardin, Nucl. Instrum. Methods Phys. Res., B 268 (2010) 223.

[22] C.S. Becquart, C. Domain, Nucl. Instrum. Methods Phys. Res., Sect. B 255 (2007) 23-26.

[23] P.E. Lhuillier et al., J. Nucl. Mater. (2010), doi:10.1016/j.jnucmat.2010.12.174. 